\title{
Proteome analysis of hepatic non-parenchymal cells of immune liver fibrosis rats
}

\author{
ZHAO QianQian ${ }^{1}$, FENG YanLing ${ }^{1}$, JIA XiaoFang ${ }^{1}$, YIN Lin ${ }^{1}$, ZHENG Ye $^{1}$, \\ OUYANG DongSheng ${ }^{2}$, ZHOU HongHao ${ }^{2 *} \&$ ZHANG LiJun ${ }^{1,2^{*}}$ \\ ${ }^{1}$ Shanghai Public Health Clinical Center, Fudan University, Shanghai 201508, China; \\ ${ }^{2}$ Institute of Clinical Pharmacology, Pharmacogenetics Research Institute, Central South University, Changsha 410078, China
}

Received May 20, 2013; accepted November 20, 2013; published online February 19, 2014

\begin{abstract}
Elucidation of the mechanisms of liver fibrogenesis is important to treat liver fibrosis. In this study, we established rat models of liver fibrosis with stages from $0-1,2$, and 3-4 to 4 at 2, 4, 6, and 8 weeks, respectively, by injection of pig serum. Liver fibrogenesis was detected by Masson's trichrome staining. Rat non-parenchymal cells (NPCs) were enriched 4-fold by Percoll density gradient centrifugation. Protein extracts from NPCs were prepared at 4 and 8 weeks, separated by two-dimensional electrophoresis, and then stained with Coomassie Blue G-250. At 4 weeks, we identified 18 non-redundant differentially expressed proteins of which protein disulfide-isomerase associated protein 3 (PDIA3) and NDUV showed consistent expression at protein and mRNA levels from 4 to 8 weeks. PDIA3 was found to be down-regulated by Western blotting in the rat model and immunohistochemically in human liver. Our results revealed important aspects of the pathogenesis/progression of liver fibrosis and demonstrated important changes in protein expression levels of NPCs at various stages of liver fibrosis.
\end{abstract}

liver fibrosis, proteomics, non-parenchymal cells, protein disulfide-isomerase associated protein 3

Citation: Zhao QQ, Feng YL, Jia XF, Yin L, Zheng Y, Ouyang DS, Zhou HH, Zhang LJ. Proteome analysis of hepatic non-parenchymal cells of immune liver fibrosis rats. Sci China Life Sci, 2014, 57: 303-314, doi: 10.1007/s11427-014-4619-0

The liver is the largest organ in the body, and liver disease has many negative effects on human health. In China, approximately 600 million people are infected with hepatitis B (HBV), of which approximately 130 million are chronic carriers of HBV and 30 million are HBV patients. Each year, approximately 300000 people do not survive liver disease, including liver cirrhosis and primary hepatocellular carcinoma, and $50 \%$ of mortalities are caused by primary hepatocellular carcinoma [1,2]. Most liver disease progresses from hepatitis, liver fibrosis and cirrhosis to liver cancer. Among these stages, only liver fibrosis can be reversed. Liver fibrosis results primarily from the action of hepatic stellate cells, non-parenchymal cells (NPCs) of the liver, which transdifferentiate into fibrogenic, proliferative, and

*Corresponding author (email: zhanglijun1221@163.com; hhzhou@public.cs.hn.cn) contractile myofibroblasts. NPCs include four main cell types, liver sinusoidal endothelial cells (LSECs), lymphocytes, Kupffer cells (KCs), and fibrocytes, which play important roles in mediation of early innate and adaptive immune responses [3-6]. However, there is limited knowledge of the proteins and pathways involved in liver fibrosis. Immune liver fibrosis (ILF) models [7-9], which mimic chronic hepatic injury caused by $\mathrm{HBV}$, have been widely used to study liver fibrosis. Pig serum-induced rat hepatic fibrosis $[7,8]$ is the most widely used model because the changes characterized by mononuclear cell infiltration and fibrotic responses in the periportal area are very similar to those in humans [8]. Thus, many researchers have used this model $[7,10]$ to study human liver fibrosis, especially that caused by HBV [11].

Proteomic technologies have high throughput, high sen- 
sitivity, and are widely applied to study liver fibrogenesis and cirrhosis development [12-16]. Most proteomic research of liver fibrosis has been performed using tissues, plasma, or cell lines. There are few studies of the proteome in primary cells, especially NPCs such as KCs $[17,18]$, LSECs [19], and hepatic stellate cells [20]. For example, Hirsch et al. [18] investigated the effect of ischemia/ reperfusion injury on the proteome of $\mathrm{KCs}$ from a rat model of ischemia/reperfusion injury, and found elevation of $\mathrm{Cu}$, $\mathrm{Zn}$-superoxide dismutase. Teufelhofer et al. [17] analyzed the proteomes of purified rat liver primary cells and blood plasma for comparison with that of liver whole tissue. Li et al. [19] reported the proteomic profile of the plasma membrane of LSECs from normal rat liver. However, no study has reported proteomic changes in the NPCs of a liver fibrosis model.

To increase our understanding of the protein changes in fibrotic liver NPCs and identify novel biomarkers to facilitate the diagnosis and treatment of liver fibrosis, we applied a quantitative proteomic approach to analyze the protein expression of NPCs from rat models with various stages of liver fibrosis and normal controls. Eight proteins were selected and analyzed by real-time reverse transcription-PCR (qRT-PCR). Among these proteins, protein disulfideisomerase associated protein 3 (PDIA3) was analyzed further by Western blotting and immunohistochemistry. To our knowledge, this is the first proteomic study of NPCs during the development of liver fibrosis.

\section{Materials and methods}

\subsection{Animal treatments}

A total of 40 eight-week-old male Sprague-Dawley rats (180-200 g) were purchased from the Center of Laboratory Animals, Shanghai Public Health Clinical Center, Shanghai, China. Animal studies were performed following the relevant national legislation and local guidelines. Establishment of the animal model was performed at the Center of Laboratory Animals according to a previously published procedure [21]. Each rat in pig serum and control groups was intraperitoneally injected with $0.5 \mathrm{~mL}$ pig serum (COSMO BIO, Japan) or $0.5 \mathrm{~mL}$ saline, respectively. At 2, 4, 6 and 8 weeks, five rats from each group were sacrificed following starvation for 16-18 h. These rats were designated as ILF-2, ILF-4, ILF-6, and ILF-8 for serum-treated rats at 2, 4, 6, and 8 weeks, respectively, and Nor- 2 , Nor- 4 , Nor- 6 , and Nor- 8 for the respective controls. The rat livers were used for histopathology and NPC separation. NPCs from rat livers at 4 and 8 weeks were used for two-dimensional electrophoresis (2DE), qRT-PCR, and Western blot analyses.

\subsection{Hepatic NPC isolation}

NPCs were isolated from liver tissue by collagenase type IV $\left(0.05 \mathrm{mg} \mathrm{mL} \mathrm{mL}^{-1}\right)$ digestion and density gradient centrifugation using Percoll (Pharmacia, Sweden) as described elsewhere [22,23] with slight modifications. Briefly, liver tissues were perfused for $10 \mathrm{~min}$ with pre-perfusion buffer followed by collagenase perfusion until digestion. The resulting suspension was filtered through a $200-\mu \mathrm{m}$ mesh and then diluted by $2 \times$ the volume with PBS. A sample containing hepatic parenchymal cells and NPCs was analyzed by flow cytometry. The remaining cell suspension was centrifuged at $100 \times g$ for $5 \mathrm{~min}$ at $20^{\circ} \mathrm{C}$. The cell pellets were resuspended and centrifuged at $400 \times \mathrm{g}$ for $10 \mathrm{~min}$ at $20^{\circ} \mathrm{C}$. The resulting cell pellet was resuspended in PBS and layered on top of $25 \%$ Percoll. The NPC fraction was enriched by density centrifugation using a $25 \%$ and $50 \%$ Percoll gradient $\left(1: 1: 1\right.$ volume ratio) at $900 \times g$ for $30 \mathrm{~min}$ at $20^{\circ} \mathrm{C}$. NPCs between the $25 \%$ Percoll layer and upper supernatant layer were collected and washed with PBS. After centrifugation at $900 \times g$ for $10 \mathrm{~min}$ at $20^{\circ} \mathrm{C}$, a small sample of the NPCs was used to verify the purity by flow cytometry, and the remaining NPCs were stored at $-80^{\circ} \mathrm{C}$ until use.

\subsection{Histopathology}

Rat liver tissue was fixed in $4 \%$ paraformaldehyde/PBS. Five-micrometer paraffin sections were subjected to Masson's trichrome staining as described previously [21]. Hepatic parenchymal cell and NPC fractions purified by Percoll density centrifugation were fixed in $4 \%$ paraformaldehyde and then stained with Masson's trichome.

\subsection{Flow cytometric analysis}

NPC purity was evaluated by flow cytometry as described previously [24]. Briefly, purified NPCs and total liver cells $\left(1 \times 10^{6}\right)$ were incubated with $1 \mu \mathrm{L}$ mouse anti-rat CD3-PE, anti-CD146-FITC, and anti-F4/80-APC for $30 \mathrm{~min}$ at room temperature in the dark. After two washes with PBS, the cells were resuspended in $3 \%$ paraformaldehyde and analyzed by a BD Diva (BD Biosciences, USA). Unstained samples were used as controls. Data analysis was performed using FlowJo software.

\subsection{DE and gel staining}

Pooled NPCs from 4- and 8-week time points were analyzed by 2DE using an IPGphor isoelectronic focusing system (GE Healthcare, USA) and Protein II electrophoresis apparatus (Bio-Rad, USA) as described previously [21,25]. A total of $1 \mathrm{mg}$ of protein from each sample was applied to IPG dry strips $(\mathrm{pH} \mathrm{3-10} \mathrm{NL,} 180 \mathrm{~mm} \times 30 \mathrm{~mm} \times 0.5 \mathrm{~mm})$. Focusing was performed at $20^{\circ} \mathrm{C}$ under the following conditions: $30 \mathrm{~V}$ for $12 \mathrm{~h}, 500 \mathrm{~V}$ for $1 \mathrm{~h}, 1000 \mathrm{~V}$ for $1 \mathrm{~h}$, an $8000 \mathrm{~V}$ gradient for $30 \mathrm{~min}$, and $8000 \mathrm{~V}$ for $6 \mathrm{~h}$ up to $52.1 \mathrm{kVh}$. The Protein II electrophoresis apparatus was used to run the $11.5 \%$ separation gels. The gels were then stained with 
Coomassie Blue G-250 as described previously [25].

\subsection{Gel image analysis and statistics}

The 2DE gels were scanned by an Imagescanner (GE Healthcare) in transmission mode. Image analysis was conducted with ImageMaster 2D Platinum (GE Healthcare). For differential analysis, fibrotic liver samples were compared with healthy control samples at the same number of weeks. Each sample was analyzed in at least three replicate gels. After spot detection, artifacts were removed by normalizing the individual spot volumes with the total optical density $(A)$ values of all spots in the gel. Differential proteomic analysis between the serum-treated and control groups was performed using the Student's $t$-test in the ImageMaster software. Differences were considered significant at $P<0.05$ and with a threshold of 2 -fold changes in expression.

\subsection{Identification of differentially expressed proteins by mass spectrometry}

After ImageMaster analysis, spots of differentially expressed proteins were cut out of the gels, digested, and then analyzed by nano flow liquid chromatograph (LC)electrospray ionization tandem mass spectrometry (MS) according to our previous reports $[21,26]$. The tryptic peptide mixtures were injected into an Ultimate 3000 instrument (LC Packings, Dionex, USA) with a C18 $\mu$-precolumn (300 $\mu \mathrm{m}$ id $\times 5$ mm, $5 \mu \mathrm{m}$, PepMap ${ }^{\mathrm{TM}}$ ) (LC Packings, Dionex, The Netherlands) at a flow rate of $20 \mu \mathrm{L} \mathrm{min}{ }^{-1}$. After desalting in the precolumn, the peptides were eluted into a C-18 reversed-phase nanocolumn $(75 \mu \mathrm{m}$ id $\times 15 \mathrm{~cm}$ length, $3 \mu \mathrm{m}$, PepMap ${ }^{\mathrm{TM}}$ ) (LC Packings) with a continuous acetonitrile gradient consisting of $4 \%-64 \%$ solvent B (B: $80 \%$ acetonitrile with $0.1 \%$ formic acid; A: $0.1 \%$ formic acid in $\mathrm{ddH}_{2} \mathrm{O}$ ) at a flow rate of $0.3 \mu \mathrm{L} \mathrm{min}{ }^{-1}$ for $40 \mathrm{~min}$. The eluted peptides were online injected using a PicoTip emitter nanospray needle (New Objective, USA) for online ionization and peptide fragmentation in an esquire HCT mass spectrometer (Bruker-Daltonics, Germany). Chromeleon 6.80 and EsquireControl 3.2 software were used to control the analytical process.

Mascot 2.2.04 was used for database searches. The search parameters were as follows: enzyme, trypsin; allowance for up to one missed cleavage peptide; mass tolerance, 1.2 Da for MS and 0.6 Da for MS/MS; fixed modification parameter, carbamidomethyl $(\mathrm{C})$; variable modification parameters, oxidation (at Met); auto hits allowed (only significant hits were reported); results formatted as a peptide summary report. Proteins were identified based on peptides with ion scores exceeding the threshold $(P<0.05)$, indicating identification at a $95 \%$ confidence level for matched peptides. Proteins identified by four or more peptides, among which at least one peptide had four or more continuous y- or b-series ions (e.g., y4, y5, y6, and y7), were accepted without manual checking. The proteins identified with three or less peptides were checked manually.

\subsection{Data analysis and bioinformatics}

The function and location of the identified proteins were elucidated using the Gene Ontology (GO) database linked to SWISS-PROT (www.expasy.org). A protein-protein interaction network was drawn by STRING 8.0 (http://string. embl.de/) using the identified proteins as search seeds.

\subsection{RNA extraction and qRT-PCR}

Total RNA from NPCs was extracted using Trizol reagent (Invitrogen, Life Technologies, USA) following the manufacturer's instructions and those described previously [19]. Briefly, qRT-PCR was performed using the iCycler iQ Multicolor Real-Time PCR Detection System (Bio-Rad) and SYBR Green (ToYoBo, Japan). For each qRT-PCR, $2 \mu \mathrm{L}$ cDNA was used with $0.7 \mu \mathrm{L}$ of $10 \mathrm{nmol} \mathrm{L}^{-1}$ sense and antisense primers (Table 1) in a total volume of $25 \mu \mathrm{L}$. The PCR conditions were $3 \mathrm{~min}$ at $95^{\circ} \mathrm{C}$, followed by 40 cycles of $95^{\circ} \mathrm{C}$ for $5 \mathrm{~s}, 60^{\circ} \mathrm{C}$ for $20 \mathrm{~s}$, and $72^{\circ} \mathrm{C}$ for $20 \mathrm{~s}$. All reactions were performed in duplicate. The threshold cycle of each qRT-PCR was converted to a DNA equivalent by reading standard curves generated by amplifying dilutions of a linearized plasmid containing $188 \mathrm{bp}$ of GAPDH cDNA. The relative quantity of target mRNA was normalized to the mRNA level of the internal control (GAPDH).

\subsection{Western blotting}

Protein extracts $(50 \mu \mathrm{g})$ of NPCs from rats at 4 and 8 weeks were separated by electrophoresis in $11.5 \%$ SDS-polyacrylamide gels and then transferred to PVDF mem-

Table 1 Sense and antisense primers for the eight selected genes

\begin{tabular}{|c|c|c|}
\hline Gene & Strand & Primer \\
\hline \multirow[t]{2}{*}{ CH60_RAT } & sense & AAAGATGGGGTCACTGTTGC \\
\hline & antisense & CATCACACCTCTCCGGATTT \\
\hline \multirow[t]{2}{*}{$H S P 7 C \_R A T$} & sense & CAGAATCCCCAAGATCCAGA \\
\hline & antisense & GTGACATCCAAGAGCAGCAA \\
\hline \multirow[t]{2}{*}{$N D U V 2 \_R A T$} & sense & TACACCTTGCATGCTTCGAG \\
\hline & antisense & TCCTTGGGTGTCAGATCCTC \\
\hline \multirow[t]{2}{*}{$O T C \_R A T$} & sense & CGTCTTCAAGCTTTCCAAGG \\
\hline & antisense & TTGCAGGCATCAGAACTTTG \\
\hline \multirow[t]{2}{*}{ PDIA3_RAT } & sense & TATGATGGGCCTAGGACTGC \\
\hline & antisense & GATTCAACGTTGGTGTGTGC \\
\hline \multirow[t]{2}{*}{$T T H Y \_R A T$} & sense & ATGGTCAAAGTCCTGGATGC \\
\hline & antisense & GCCAAGAGCCTTCCAGTATG \\
\hline \multirow[t]{2}{*}{$U C R I \_R A T$} & sense & AGGGGAAGAACATGGCTTTT \\
\hline & antisense & GGGCAATAGTAGCCACCAAA \\
\hline \multirow[t]{2}{*}{$A T P B \_R A T$} & sense & CAGGCTGGCTCAGAGGTATC \\
\hline & antisense & ATGGGCAAAGGTAGTTGCAG \\
\hline
\end{tabular}


branes (Millipore, USA). The blots were incubated overnight at $4{ }^{\circ} \mathrm{C}$ with the following primary antibodies: antiPDIA3 (1:1000; ProteinTech, USA) and rabbit antiprohibitin (1:1000; Abcam, Hong Kong, China). After three washes with TBS-Tween, the blots were incubated for $1 \mathrm{~h}$ at $20^{\circ} \mathrm{C}$ with secondary antibodies. Immune complexes were detected by enhanced chemiluminescence and exposure to $\mathrm{X}$-ray film.

\subsection{Immunohistochemical analysis of PDIA3 in hu- man liver tissue}

Immunohistochemistry was performed as described previously [20]. Briefly, tissue sections were incubated for $1 \mathrm{~h}$ at room temperature with a rabbit anti-PDIA3 antibody $(1: 100)$ and then a horseradish peroxidase-conjugated anti-rabbit antibody. The signals were detected using a liquid 3,30-diaminobenzidine staining kit (Gene Tech, USA). Images were obtained under a BX40 microscope (Olympus, Japan) equipped with a logenE PAS9000 at $100 \times$ or $400 \times$ magnification. To semi-quantitatively analyze the expression of PDIA3 in liver NPCs, each section was randomly imaged five times in the domains of NPCs at $400 \times$ magnification. PDIA3-positive cells were then counted in each image. The ratio of the average number of positive cells from 20 patients at stage (S) 3-4 (100 images) to that of 20 patients at S0-1 (100 images) was considered as the rate of PDIA3 expression changes.

\section{Results}

\subsection{Histopathological findings}

We established the ILF model according to our previous study [21]. As shown in Figure S1 in Supporting Information, at 2 weeks after serum injection, inflammatory cell infiltration and hepatic fibrosis occurred around the portal area, and liver fibrosis was diagnosed as S0-1. At 4 weeks post-injection, the liver fibrosis had developed to S2. At 6 weeks, the rat liver was thin, contained separated and small pseudolobules, and was diagnosed as S2, S3, or S3-4. At 8 weeks, very thick pseudolobules had developed, and the diagnosis was S4. In contrast, livers in the control group showed no histopathological changes during the experimental period.

\subsection{NPC enrichment}

To analyze the enrichment of NPCs, we performed hematoxylin and eosin $(\mathrm{H} \& \mathrm{E})$ staining and flow cytometric detection of CD146, F4/80, and CD3, which are specific biomarkers of LSECs, KCs, and lymphocytes, respectively. In the purified NPCs, H\&E staining showed that contamination of red blood cells and hepatocytes was low (Figure
S2A-D in Supporting Information). As shown in Figure S2B in Supporting Information, hepatic parenchymal cells were absent. Among the images of H\&E staining, we found only one liver parenchymal cell. The proportion of red blood cells was $11.84 \%$ in the purified NPC fraction (Figure S2C and D in Supporting Information). These results indicated successful separation of the hepatic NPCs by Percoll density gradient centrifugation.

To quantitatively analyze the enrichment of NPCs, CD3-, F4/80-, and CD146-positive cells (representing lymphocytes, $\mathrm{KCs}$, and LSECs, respectively) were quantified by flow cytometry. Unstained cells were used as controls (Figure S3 in Supporting Information; Figure 1). In the NPC population, CD3-, F4/80-, and CD146-positive cells were $30.10 \% \pm 0.20 \%, 8.04 \% \pm 0.50 \%$, and $41.17 \% \pm 0.49 \%$, and $19.07 \% \pm 0.35 \%, 2.52 \% \pm 0.61$, and $11.00 \% \pm 0.60 \%$ in total liver cells, respectively. Compared with the total liver cells, lymphocytes, KCs, and LSECs were enriched by more than 1.5-fold (Figure 1A and B), 3.2-fold (Figure 1C and D), and 3.7-fold (Figure 1E and F) in NPCs, respectively.

\section{$2.3 \quad 2-D E$ and gel image analysis}

Proteins of the ILF models and controls were separated using $\mathrm{pH} 3-10 \mathrm{NL}$ gel strips in the first dimension and an $11.5 \%$ separation gel in the second dimension. Similar protein spot profiles were obtained from ILF and corresponding control samples. In the ImageMaster analysis, the spot numbers of detectable proteins in the Coomassie Bluestained gels were $637 \pm 47,608 \pm 43,639 \pm 52$, and $602 \pm 36$ in ILF samples at 4 weeks, normal samples at 4 weeks, ILF samples at 8 weeks, and normal samples at 8 weeks, respectively. The raw data of the ImageMaster analysis, including intensity values, volumes, volume\% (after normalization), area of identified differential spots, and the numbers of matched protein spots, are provided in Table S1 in Supporting Information. The changes at the various time points were less than those between the ILF model and controls according to the protein spots in 2DE (Figure 2; Figure S4 in Supporting Information). Based on the average intensity ratios of protein spots, a total of 48 protein spots were dynamically changed during the development of liver fibrosis with 22 and 26 differentially expressed proteins at 4 and 8 weeks, respectively. Among these proteins, 12 and 14 up-regulated protein spots were detected at 4 and 8 weeks (ILF/normal ratios of $\geqslant 2, P \leqslant 0.05$ ), respectively (data not shown). Some of these differentially expressed proteins showed consistent regulation, such as spots 1 and $1^{*}, 3$ and $3^{*}$, and 14 and 14* (Figure 2; Figure S4 in Supporting Information). Based on the partially magnified images of spots 14 and $14^{*}$ (Figure 3A), significant down-regulation was found in all gels from ILF models compared with those from the controls at 4 and 8 weeks. However, some proteins with inconsistent regulation, such as spots 2, 5 and 8, were up-regulated in ILF models at 4 weeks but down-regulated 



Figure 1 Flow cytometric analysis of CD3-, CD146-, and F4/80-positive cells. NPCs and total liver cells were stained with anti-CD3, -CD146- and -F4/80 antibodies. Unstained cells were used as the negative control. A and B, C and D, and E and F represent flow cytometry analyses of CD3-, F4/80-, and CD146-positive cells among NPCs and total liver cells, respectively. The number of cells in a representative experiment is shown in the panels.

at 8 weeks (Figure 2C and D, indicated as $2 \mathrm{x}, 5 \mathrm{x}$, and $8 \mathrm{x}$, respectively). Similarly, spot 10 , a down-regulated protein in ILF models at 4 weeks, was up-regulated in ILF models at 8 weeks. To identify the proteins related to liver fibrosis, only the differential protein spots in $2 \mathrm{DE}$ at 4 weeks were recovered and identified by MS. The gels at 8 weeks were used to check the dynamic regulation of the differentially expressed proteins detected at 4 weeks.

\subsection{Identification of differentially expressed proteins}

Using MS, we identified 18 of the 22 differential protein spots with greater than 2-fold expression changes, including eight up-regulated and 10 down-regulated proteins in ILF 

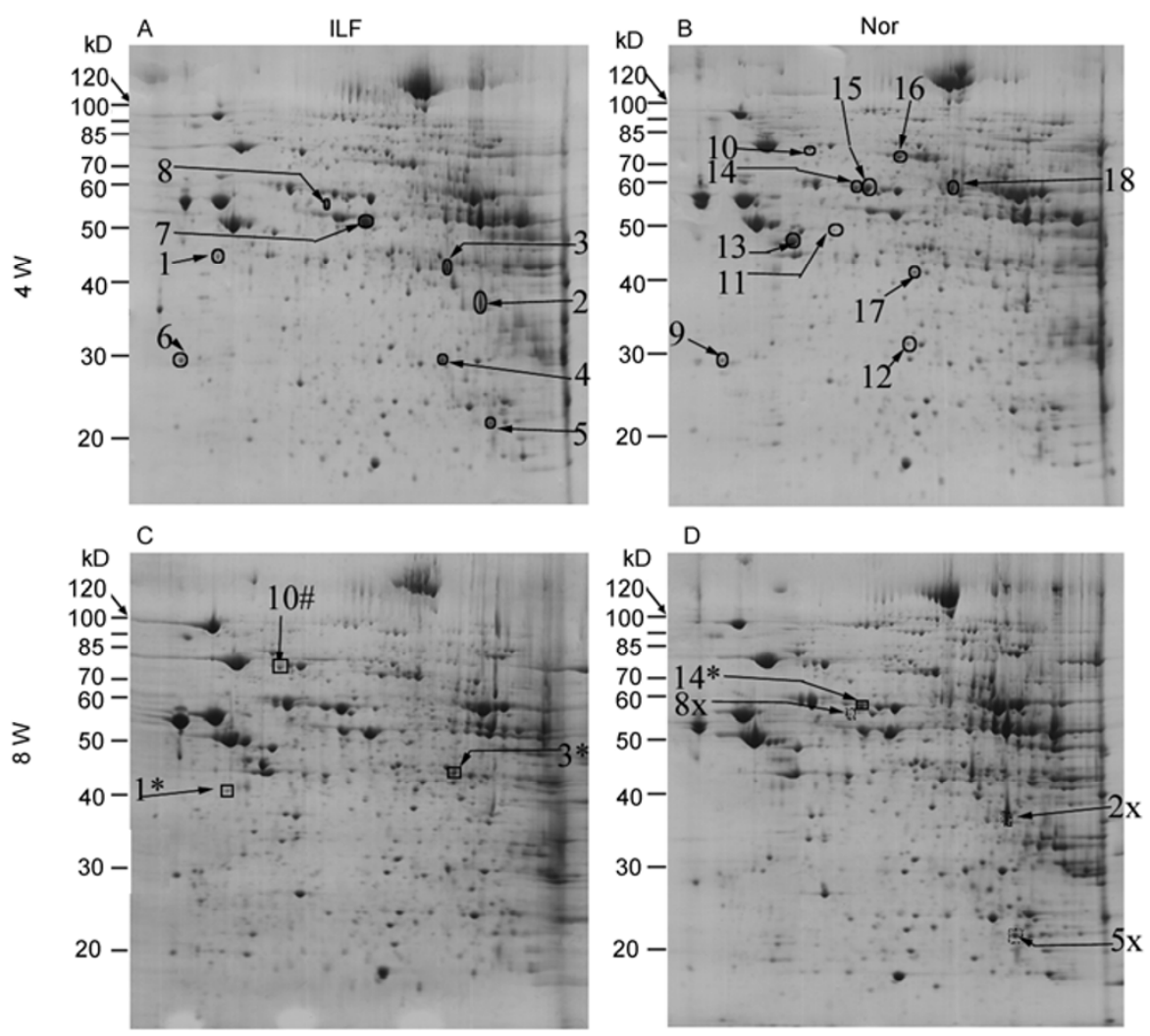

Figure 2 Protein expression profiles of ILF models and normal controls. ILF, immune liver fibrosis; Nor, normal control. Samples from serum- or saline-treated rats at 4 and 8 weeks are represented by $4 \mathrm{~W}$ and $8 \mathrm{~W}$, respectively. Up-regulated proteins are labeled in the ILF gel (A), and down-regulated proteins are marked in the normal gel (B). Proteins with consistent mRNA and protein levels continually up- or down-regulated in samples from 4 to 8 weeks $(\mathrm{C}$ and $\mathrm{D})$ are marked with $1^{*}, 3^{*}$, and $14^{*}$. The proteins with consistent mRNA and protein levels but opposing expression in samples at 4 and 8 weeks are labeled with 10\#. Proteins with inconsistent mRNA and protein levels are indicated by $2 x, 5 x$, and $8 x$.

models compared with those in normal controls at 4 weeks (Table 2). Among these 18 proteins, only one was identified by one peptide with a Mascot score of 47 (Table S2, Figure S5 in Supporting Information). Each protein had at least one peptide with continuous four $\mathrm{y}$ or $\mathrm{b}$ ions by manual checking. For example, in protein disulfide-isomerase A3 (spot 14), $40 \%$ of the amino acids were accurately detected by MS with 22 matched non-redundant peptides (Figure 3B, underlines). Based on the representative MS/MS spectra of peptide DLLTAYYDVDYEK, almost all b or y ions were detected by MS for disulfide-isomerase A3 (Figure 3C). The abundance of these differentially expressed proteins was low according to the data from exponentially modified protein abundance index protein (emPAI) analysis, including 14 with an emPAI of $<1.0$ (Table 2; Table S2 in Supporting Information).

\subsection{Bioinformatic analysis of differentially expressed proteins}

To determine the location and function of these proteins, we used the UniProt knowledgebase (Swiss-Prot/TrEMBL) and GO database. The differentially expressed proteins were widely distributed in all types of subcellular organelles, including seven in mitochondria, three in the plasma membrane, three in the cytoplasm, three in the endoplasmic reticulum (ER), and others (Table 2). These proteins have important functions such as binding (including ATP, DNA, and actin binding) (50\%), enzymatic activity (38\%), electron carriers (8\%), and others (Table 2).

NPCs perform important functions in liver fibrosis. The proteins in NPCs interact with each other and also communicate with other hepatic cells. Thus, in this study, a protein-protein interaction network was created to determine the interactions of these differentially expressed proteins with each other. For 10 proteins, interactions were detected with other proteins, but for seven proteins, no interactions were detected in this analysis (Figure S6 in Supporting Information). These interactive proteins included Uqcrfs1, Ndufv2, and ATP5B, which interact with each other. In addition, Hspa8 interacts with CH60, CH60 with Pdia3, Ttr with Alb, and Aldh2 with Otc. Some of these interactive proteins had a consistent trend of protein expression, such as Ndufv2, ATP5B and Uqcrfs1, which were up-regulated in the ILF model, but others showed an opposing expression trend, such as PDIA3 and its protein partner CH60, which were down- and up-regulated, respectively. Among these differentially expressed proteins, HSP7C, NDUV, PDIA3, 

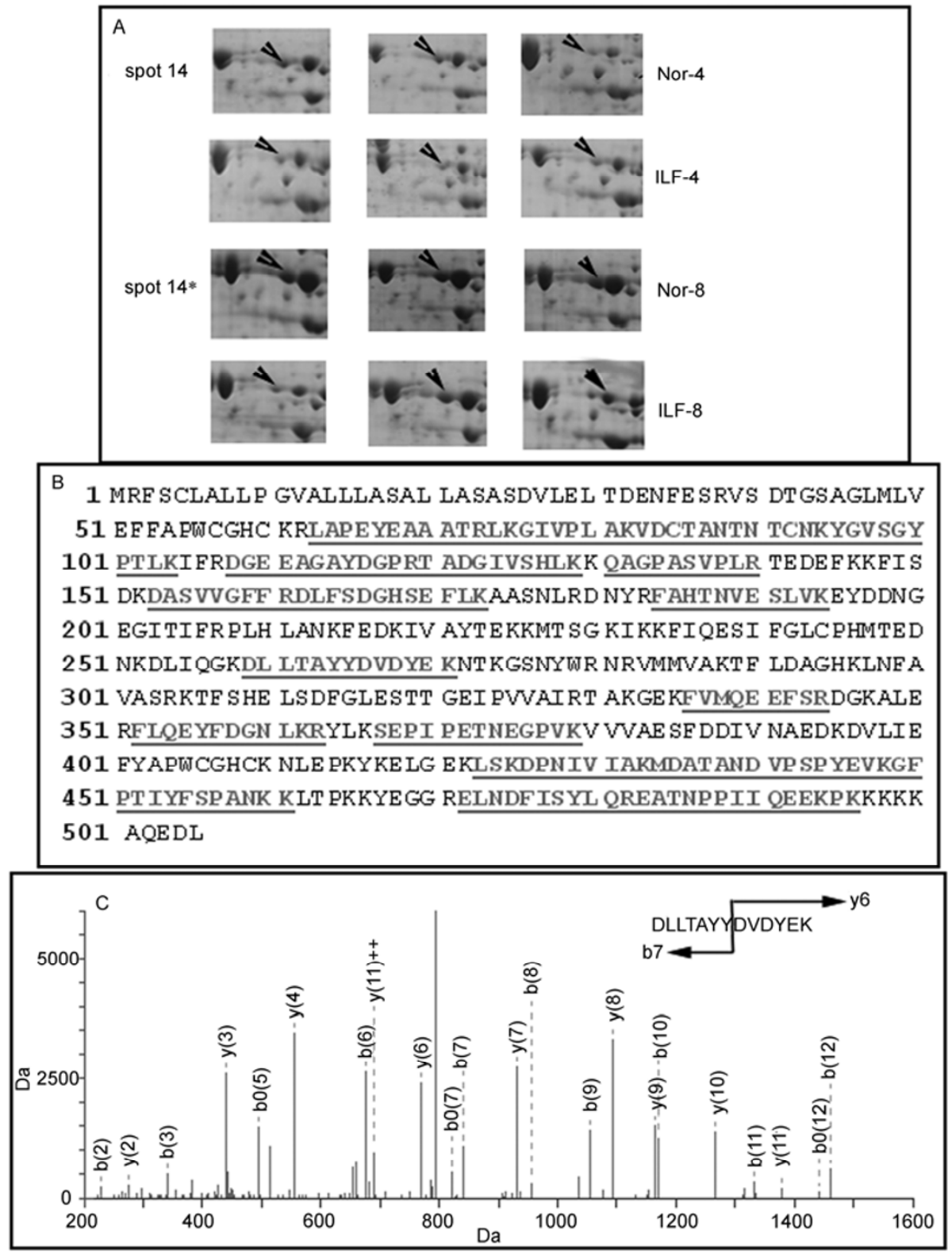

Figure 3 Representative enlarged 2DE gels and MS/MS protein identification results. A, Partially enlarged gel profiles for spot 14. B, Sequence coverage of spot 14. Underlined peptides were detected by MS. C, Representative MS/MS spectrum of peptide DGEEAGAYDGPR from spot 14.

OTC, UCRI, TTHY, ATPB, and CH60 were located in nodes of the network and were thus chosen for qRT-PCR analysis.

\subsection{Transcriptional profiles of differentially expressed proteins}

To determine whether the protein expression changes occur at transcriptional or translational levels, the transcriptional alterations of eight selected genes in NPCs from ILF and control rats were measured by qRT-PCR (Figure S7 in Supporting Information). Among them, five genes showed mostly consistent expression at protein and mRNA levels, including PDIA3, TTHY, NDUV, ATPD, and CH60. PDIA3 and TTHY expression was down-regulated at 4 and 8 weeks compared with that in the corresponding controls, and the degree of down-regulation decreased from 4 to 8 weeks (Tables 3, Figure 4; Table S3 in Supporting Information). mRNAs of NDVU, ATPB, and CH60 were up-regulated at both 4 and 8 weeks compared with those in the corresponding controls. Among these five differentially expressed genes, only PDIA3 and NDUV showed completely consistent expression at protein and mRNA levels from 4 to 8 weeks. 
Table 2 Differential protein spots in 2DE from ILF models and normal controls at 4 weeks

\begin{tabular}{|c|c|c|c|c|c|c|c|c|c|c|c|}
\hline Spot $^{\text {a) }}$ & No. ${ }^{\text {b) }}$ & Protein & Score $^{\text {c) }}$ & $\mathrm{pI}^{\mathrm{d})}$ & $\mathrm{MW}^{\mathrm{e})}$ & Cov. ${ }^{f)}$ & $\mathrm{Reg}^{\mathrm{g})}$ & emPAI & Location $^{\text {h) }}$ & Function ${ }^{\text {i) }}$ & Interaction ${ }^{\mathrm{j})}$ \\
\hline 1 & АТPB & $\begin{array}{l}\text { ATP synthase subunit beta, } \\
\text { mitochondrial }\end{array}$ & 409 & 5.19 & 56318 & $19 \%$ & 4.75 & 0.62 & Mit. & $\begin{array}{l}\text { binding/ } \\
\text { enzyme }\end{array}$ & ATP5B \\
\hline 2 & OTC & $\begin{array}{l}\text { Ornithine carbamoyltrans- } \\
\text { ferase, mitochondrial }\end{array}$ & 393 & 9.12 & 39919 & $32 \%$ & 2.45 & 0.98 & Mit. & $\begin{array}{l}\text { binding/ } \\
\text { enzyme }\end{array}$ & Otc \\
\hline 3 & NDUV2 & $\begin{array}{l}\text { NADH dehydrogenase } \\
\text { [ubiquinone] flavoprotein } \\
2 \text {, mitochondrial }\end{array}$ & 95 & 6.23 & 27709 & $18 \%$ & 4.67 & 0.31 & Mit. & $\begin{array}{l}\text { electron carrier/ } \\
\text { binding }\end{array}$ & Ndufv2 \\
\hline 4 & ECH1 & $\begin{array}{c}\text { Del- } \\
\text { ta(3,5)-Delta(2,4)-dienoyl- } \\
\text { CoA isomerase, mitochon- } \\
\text { drial }\end{array}$ & 389 & 8.13 & 36496 & $26 \%$ & 8.50 & 0.73 & Mit. & $\begin{array}{l}\text { binding/ } \\
\text { enzyme }\end{array}$ & Ech1 \\
\hline 5 & UCRI & $\begin{array}{l}\text { Cytochrome b-c1 complex } \\
\text { subunit Rieske, mitochon- } \\
\text { drial }\end{array}$ & 157 & 9.04 & 29717 & $8 \%$ & 6.85 & 0.46 & PM & $\begin{array}{l}\text { electron carrier/ } \\
\text { binding/ } \\
\text { enzyme }\end{array}$ & Uqcrfs1 \\
\hline 6 & C1QBP & $\begin{array}{l}\text { Complement component } 1 \\
\text { Q subcomponent-binding } \\
\text { protein, mitochondrial }\end{array}$ & 388 & 4.77 & 31326 & $21 \%$ & 3.49 & 0.56 & Mit. & binding & C1qbp \\
\hline 7 & ALDH2 & $\begin{array}{l}\text { Aldehyde dehydrogenase, } \\
\text { mitochondrial }\end{array}$ & 1065 & 6.63 & 56975 & $42 \%$ & 2.30 & 2.15 & Mit. & enzyme & Aldh2 \\
\hline 8 & CH60 & $\begin{array}{l}\text { 60-kDa heat shock protein, } \\
\text { mitochondrial }\end{array}$ & 500 & 5.91 & 61091 & $30 \%$ & 5.40 & 0.75 & Mit. & $\begin{array}{l}\text { binding/ } \\
\text { enzyme }\end{array}$ & CH60 \\
\hline 9 & TPM4 & $\begin{array}{l}\text { Tropomyosin alpha- } 4 \\
\text { chain }\end{array}$ & 273 & 4.66 & 28550 & $13 \%$ & 0.40 & 0.3 & Cyt. & binding & Tpm4 \\
\hline 10 & HSP7C & Heat shock cognate & 208 & 5.37 & 71059 & $13 \%$ & 0.49 & 0.32 & Cyt. & stress response & Hspa8 \\
\hline 11 & GUAD & Guanine deaminase & 171 & 5.56 & 51564 & $14 \%$ & 0.41 & 0.22 & Unknown & enzyme & Gda \\
\hline 12 & ANXA3 & Annexin A3 & 235 & 5.96 & 36573 & $21 \%$ & 0.44 & 0.59 & PM & binding & Anxa3 \\
\hline 13 & ACTG & Actin, cytoplasmic 2 & 505 & 5.31 & 42114 & $34 \%$ & 0.21 & 1.48 & PM/cyt. & binding & Actg 1 \\
\hline 14 & PDIA3 & $\begin{array}{c}\text { Protein disulfide-isomerase } \\
\text { A3 }\end{array}$ & 1630 & 5.88 & 57052 & $40 \%$ & 0.49 & 1.65 & ER & $\begin{array}{l}\text { enzyme/ } \\
\text { binding }\end{array}$ & Pdia3 \\
\hline 15 & PDIA3 & $\begin{array}{l}\text { Protein disulfide-isomerase } \\
\text { A3 }\end{array}$ & 71 & 5.88 & 57052 & $9 \%$ & 0.43 & 0.1 & ER & $\begin{array}{l}\text { enzyme/ } \\
\text { binding }\end{array}$ & Pdia3 \\
\hline 16 & ALBU & Serum albumin & 608 & 6.09 & 70716 & $28 \%$ & 0.24 & 1.71 & PM/EXP & binding & Alb \\
\hline 17 & TTHY & Transthyretin & 209 & 5.77 & 15826 & $30 \%$ & 0.36 & 0.83 & EXP. & binding & Ttr \\
\hline 18 & CES3 & Carboxylesterase 3 & 47 & 6.1 & 62398 & $1 \%$ & 0.07 & 0.05 & ER & enzyme & Ces3 \\
\hline
\end{tabular}

a) Spot No. is a unique number that refers to the labels in Figure 2. b) No., accession numbers obtained from the SWISS-PROT database. c) Mascot score. d) Theoretical isoelectric point (pI). e) Molecular weight predicted from database. f) Sequence coverage (\%) indicates the number of amino acids spanned by the assigned peptides divided by the sequence length. g) Protein expression change in ILF models compared with that in normal controls. h) Protein location from the GO database. Mit, mitochondrial; Cyt, cytoplasm; PM, plasma membrane (including cytoskeleton, basement membrane, and integral to the membrane); ER, endoplasmic reticulum; EXP, extracellular space; Unknown, no subcellular location information was provided by the GO database. i) Protein function from the GO database. j) Abbreviation of differential proteins shown in the protein-protein interaction network.

Table 3 Comparison of the mRNA and protein levels of eight selected differentially expressed proteins in ILF rats and controls

\begin{tabular}{|c|c|c|c|c|c|}
\hline Spots & Name & $\begin{array}{c}\text { Protein } \\
4 \mathrm{~W}^{\mathrm{a})} \\
\end{array}$ & $\begin{array}{c}\text { Protein } \\
8 \mathrm{~W}^{\mathrm{a})} \\
\end{array}$ & $\begin{array}{c}\text { mRNA } \\
4 W^{b)}\end{array}$ & $\begin{array}{c}\text { mRNA } \\
8 \mathrm{~W}^{\mathrm{b})}\end{array}$ \\
\hline 14 & PDIA3 & 0.30 & 0.49 & 0.01 & 0.32 \\
\hline 17 & TTHY & 0.34 & Not detected ${ }^{\text {c) }}$ & 0.015 & 0.185 \\
\hline 3 & NDUV & 4.4 & 2.91 & 2.15 & 1.365 \\
\hline 1 & АТРВ & 8.05 & 14.6 & 1.345 & 2.91 \\
\hline 8 & CH60 & 6.37 & No change ${ }^{\text {d) }}$ & 2.015 & 2.555 \\
\hline 10 & HSP7C & 0.40 & 2.23 & 0.13 & 3.47 \\
\hline 2 & отC & 2.0 & 0.29 & 0.025 & 1.21 \\
\hline 5 & UCRI & 4.76 & 0.63 & 0.005 & 1.9 \\
\hline
\end{tabular}

a) $4 \mathrm{~W}$ and $8 \mathrm{~W}$ indicate rats at 4 and 8 weeks, respectively. The values are the ratios of protein expression in ILF rats compared with that in normal controls. b) Values are the ratios of mRNA expression in ILF rats compared with that in normal controls. c) Spot 17, not detected in gels for samples at 8 weeks. d) Spot 8 , no change was detected in samples at 8 weeks. 




Figure 4 mRNA expression of eight genes selected from 18 differentially expressed proteins $(P<0.05)$. TTHY-4 and TTHY- 8 represent the ratio of serum-treated to saline-treated groups at 4 and 8 weeks, respectively. GAPDH was used as an internal control. For the normal control, the value was set as "1" and marked with a line.

\subsection{Verification of PDIA3 expression by Western blotting}

The dynamic changes of one differentially expressed protein, PDIA3, were validated by Western blot analyses of NPCs from the rat ILF model. As shown in Figure 5, PDIA3 expression was significantly decreased at 4 and 8 weeks in the NPCs of serum-treated rats compared with that in the controls (Figure 5C), which was consistent with the results from 2DE analyses. Furthermore, the samples used for Western blotting were checked for consistent protein loading by the reference protein (prohibitin) (Figure 5B) and Coomassie blue staining (Figure 5A).

\subsection{Immunohistochemical analysis of PDIA3 in human liver}

To verify the findings in the rat model, we performed im-
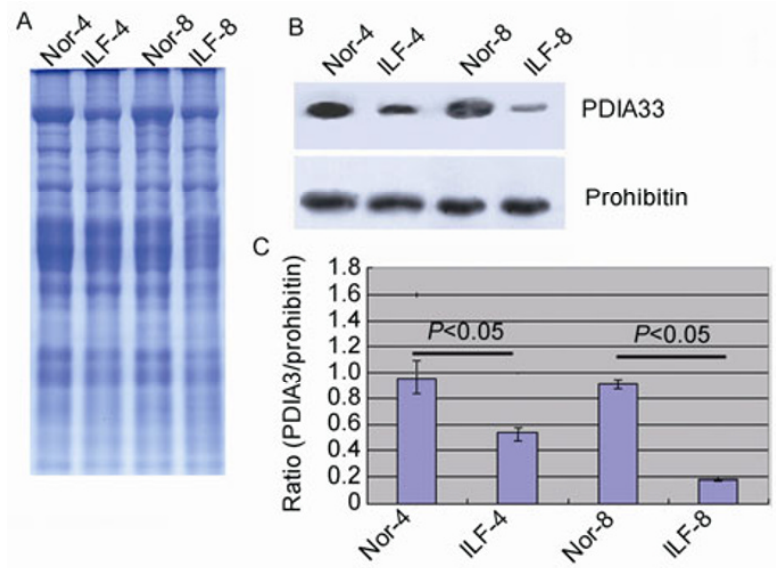

Figure 5 Western blot analysis of PDIA3. A, SDS-polyacrylamide electrophoresis. B, Expression of PDIA3 and prohibitin in rat ILF models at 4 and 8 weeks. C, Signal intensities of specific bands. The $y$-axis shows the $A$ of protein expression. Data are presented as the means and standard deviation $(n=3)$. $P<0.05$, Student's $t$-test. Nor-4, ILF-4, Nor- 8 , and ILF-8 represent hepatic protein extracts of NPCs from saline- and serum-treated groups at 4 and 8 weeks, respectively. munohistochemical analysis of human liver biopsies at S0-1 and S3-4 using an anti-PDIA3 antibody. We found a high correlation between the immunohistochemical staining and proteome expression profiles obtained by 2-DE. In S0-1 samples, the positive signals (Figure 6A and B) were stronger than those in S3-4 samples (Figure 6C and D). Statistical analysis showed that the expression of PDIA3 was decreased by 2 -fold at S3-4 $(n=20 \times 5)$ compared with that at S0-1 $(n=20 \times 5)$ (Figure $6 \mathrm{E})$.
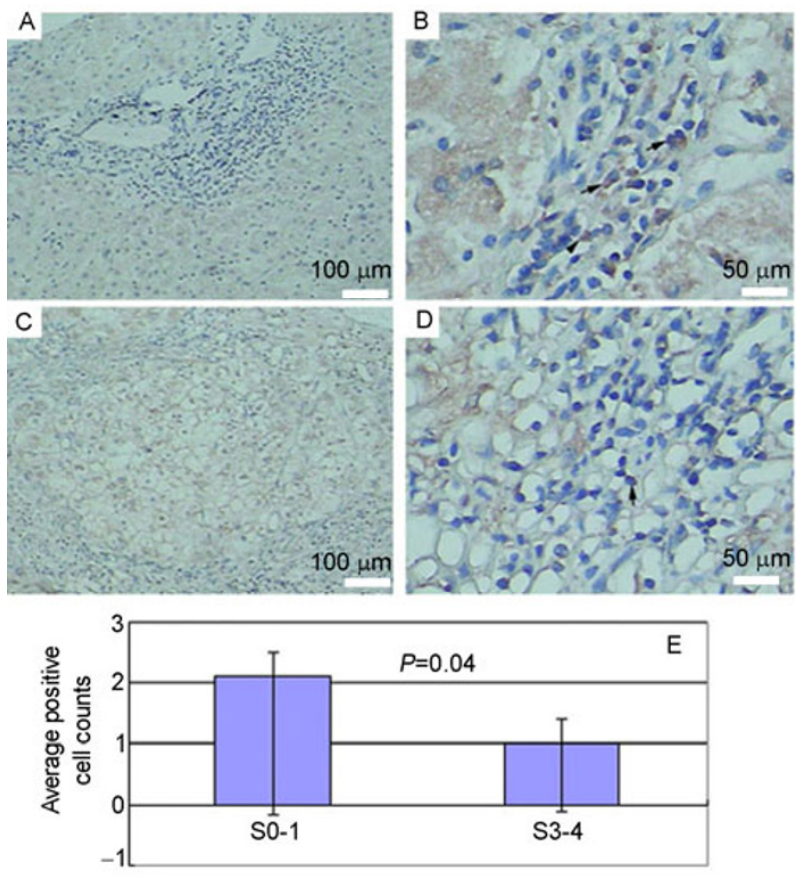

Figure 6 Immunohistochemical analysis of PDIA3. A-D, Human liver tissues at S0-1 (100×) (A), S0-1 (400×) (B), S3-4 (100×) (C), and S3-4 (400x) (D). E, Statistical analysis of average PDIA3-positive NPC counts in each image. Twenty liver tissue samples from patients at S0-1 and 20 samples at S3-4 were analyzed immunohistochemically. Each section was randomly imaged five times at $400 \times$ magnification in the domains of NPCs. $P=0.04$, S0-1 $(n=100)$, S3-4 $(n=100)$. 


\section{Discussion}

Rats develop liver fibrosis by treatment with pig serum and are thus widely used as a model to study $\operatorname{ILF}[7,8]$. In this study, liver tissues from ILF rat models at 2, 4, 6, and 8 weeks showed ILF at S0-1, S2, S2-4, and S4, respectively. At 6 weeks, it is unknown why some rats showed ILF characters of 4 or 8 weeks. Furthermore, S2 and S4 clinically represent serious liver fibrosis (liver cirrhosis with ascites). Therefore, to detect proteins related to liver fibrosis development, liver tissues at S2 and S4 were selected for $2 \mathrm{DE}$ analysis.

In this study, we analyzed NPCs from liver tissue. Usually, researches focus on hepatocytes because of their high numbers and important functions in the liver. However, NPCs also have very important functions such as immune and mediation functions in the liver [3-6]. The mechanisms regulating the responses of NPCs in liver fibrosis are unclear. Moreover, differentially expressed proteins might play critical roles in the functional changes of NPCs during the progression of liver fibrosis, and may be important to understand such mechanisms. In addition, proteomic profiling of NPCs can identify biomarkers that may serve as diagnostic and prognostic indicators as well as drug targets for liver diseases. However, in the liver, the number of NPCs is much less than that of hepatic parenchymal cells. Thus, identification of proteins in NPCs, especially those with low abundance, is difficult in proteomic analyses of whole tissues.

To overcome this problem and identify proteins related to liver fibrogenesis in NPCs, we purified NPCs using Percoll density gradient centrifugation. As shown by H\&E staining, lymphocytes, LSECs, and KCs were mainly enriched in the NPC fraction. To characterize the enrichment of NPCs, CD3-, CD146-, and F4/80-positive cells were analyzed to identify lymphocytes, LSECs and $\mathrm{KCs}$, respectively. Liver parenchymal cells do not express CD3, CD146, or F4/80 [27,28]. Although we detected enrichment of CD3-, CD146-, and F4/80-positive cells, we did not identify all of the NPCs. To our knowledge, it is very challenging and unnecessary to characterize all NPCs using biomarkers because we purified NPCs using the same method for both the ILF model and control at 4 and 8 weeks. Furthermore, proteins in a small number of cells might not be detected by proteomic approaches.

In this study, the proteome profiles of NPCs from rat livers were obtained by 2D gels stained with Coomassie Blue. Approximately 700 protein spots were separated, and 18 differentially expressed proteins were identified by MS.

To determine the concentrations of the differentially expressed proteins, we used a MS-based approach known as emPAI. This technique reveals the protein concentrations based on the correlation between the number of identified peptides and the protein abundance in MS-based proteomic experiments $[29,30]$. Here, the emPAI data were obtained from protein identification results that were acquired by Mascot database search engines. Among the differentially expressed proteins, 14 had an emPAI of $<0.98$, which are related to ornithine carbamoyltransferase with concentrations of $8-20 \mu \mathrm{g} \mathrm{L}^{-1}$ in human plasma. Thus, most of the differentially expressed proteins have medium or low abundance in liver tissue.

Liver fibrogenesis is progressive and involves the regulation of many proteins. Some proteins may be continuously up- or down-regulated, while others may have different regulatory patterns during fibrogenesis. To dynamically observe the protein profiles during liver fibrogenesis, proteins from the liver at S2 (4 weeks) and S4 (8 weeks) were separated by $2 \mathrm{DE}$. As a result, we identified 18 differentially expressed proteins.

Functional analyses revealed that these 18 differentially expressed proteins were mainly involved in binding to actin, ATP, or DNA, or enzymatic activity. These observations may be because of the natural characteristics of proteins that bind to other proteins, catalyze biochemical reactions, and metabolize all types of materials. A subcellular location analysis revealed that seven of the 18 proteins are located in mitochondria, including ATPB, OTC, NDUV2, ECH1, C1QBP, ALDH2, and CH60. This phenomenon of mostly regulated mitochondrial proteins is consistent with the theory that mitochondrial dysfunction acts as a common pathogenic mechanism in chronic liver diseases. Dysfunctional and/or uncoupled mitochondria enhance the susceptibility of hepatocytes to cell death by necrosis via ATP depletion or apoptosis via membrane permeabilization [31]. To further study the pathways regulated during this process, we constructed a protein-protein interaction network. Of the 18 differentially expressed proteins, seven proteins did not interact with the others. This result is mainly because of two reasons: only NPCs were analyzed in this study, and only proteins with high abundance can be detected by proteomic approaches.

In this study, mRNA analysis of eight differentially expressed proteins revealed that five of the eight genes were consistently up- or down-regulated from S2 to S4 with ATPB, NDUV2, and CH60 showing up-regulation and PDIA3 and TTHY showing down-regulation. ATPB, NDUV2, and PDIA3 were consistently regulated from S2 to $\mathrm{S} 4$ at mRNA and protein levels.

PDIA3 was verified to be down-regulated in the rat ILF model at $\mathrm{S} 4$ compared with that at $\mathrm{S} 2$ by Western blot analyses. Consistent down-regulation was detected in human liver tissue at S3-4 compared with that at S0-1. Similar results were obtained in a report by Aroor et al., in which the protein levels of the basic form of PDIA3 were significantly decreased after a chronic ethanol binge [32]. PDIA3 catalyzes the rearrangement of S-S bonds in proteins and has very important functions such as electron carrier and cysteine-type endopeptidase activities. Our results might 
complement or support the theory that mitochondrial dysfunction, especially in the respiratory chain, can induce liver fibrosis [33]. Furthermore, PDIA3 might serve as a new target to treat liver fibrosis [34].

In conclusion, our proteomic analyses of NPCs in fibrotic livers and normal controls by 2DE-MS identified a number of proteins related to liver fibrosis. We identified three genes (PDIA3, ATPB, and NDUV2) with consistent mRNA and protein regulation from S2 to S4. PDIA3 was verified as down-regulated in liver fibrosis at $\mathrm{S} 4$ compared with that at $\mathrm{S} 2$ by western blot analysis of the rat ILF model at 4 and 8 weeks and immunohistochemically in human liver at S0-1 to S3-4. We anticipate that further study of PDIA3 will expand our understanding of the mechanisms of liver fibrogenesis and assist in the development of new therapeutic approaches against liver fibrosis.

This work was supported by the Major New Drug Discovery Science and Technology (2012ZX09303013), the National Basic Research Program of China (2011CB910700), National Natural Science Foundation of China (81271834), China Postdoctoral Science Foundation (20100471238), China Postdoctoral Science Special Foundation (201104516), National Key Technology R\&D Program of China (2012ZX10001003), and the Postdoctoral Science Foundation of Central South University, Science and Technology Commission of Shanghai Municipality (11DZ2292900).

1 Yuen MF, Hou JL, Chutaputti A. Hepatocellular carcinoma in the Asia pacific region. J Gastroenterol Hepatol, 2009, 24: 346-353

2 Shen LP, Zhang Y, Wang F, Zhang S, Yang JY, Fang KX, Yu T, Wang XY, Zhang WY, Bi SL. Epidemiological changes in hepatitis $B$ prevalence in an entire population after 20 years of the universal HBV vaccination programme. Epidemiol Infect, 139: 1159-1165

3 Knolle PA, Gerken G. Local control of the immune response in the liver. Immunol Rev, 2000, 174: 21-34

4 Kimura K, Kakimi K, Wieland S, Guidotti LG, Chisari FV. Activated intrahepatic antigen-presenting cells inhibit hepatitis $\mathrm{B}$ virus replication in the liver of transgenic mice. J Immunol, 2002, 169: 5188-5195

5 Ramadori G, Moriconi F, Malik I, Dudas J. Physiology and pathophysiology of liver inflammation, damage and repair. J Physiol Pharmacol, 2008, 59(Suppl 1): 107-117

6 Wu J, Meng Z, Jiang M, Pei R, Trippler M, Broering R, Bucchi A, Sowa JP, Dittmer U, Yang D, Roggendorf M, Gerken G, Lu M, Schlaak JF. Hepatitis B virus suppresses toll-like receptor-mediated innate immune responses in murine parenchymal and nonparenchymal liver cells. Hepatology, 2009, 49: 1132-1140

7 Baba Y, Uetsuka K, Nakayama H, Dot K. Rat strain differences in the early stage of porcine-serum-induced hepatic fibrosis. Exp Toxicol Pathol, 2004, 55: 325-330

8 Tsukamoto H, Matsuoka M, French SW. Experimental models of hepatic fibrosis: a review. Semin Liver Dis, 1990, 10: 56-65

9 Schuppan D, Ruehl M, Somasundaram R, Hahn EG. Matrix as a modulator of hepatic fibrogenesis. Semin Liver Dis, 2001, 21: 351-372

10 Fujisawa G, Muto S, Okada K, Kusano E, Ishibashi S. Mineralocorticoid receptor antagonist spironolactone prevents pig serum-induced hepatic fibrosis in rats. Transl Res, 2006, 148: 149-156

11 Villeneuve JP. The natural history of chronic hepatitis B virus infection. J Clin Virol, 2005, 34(Suppl 1): S139-142

12 Smyth R, Lane CS, Ashiq R, Turton JA, Clarke CJ, Dare TO, York MJ, Griffiths W, Munday MR. Proteomic investigation of urinary markers of carbon-tetrachloride-induced hepatic fibrosis in the Hanover Wistar rat. Cell Biol Toxicol, 2009, 25: 499-512
13 Spano D, Cimmino F, Capasso M, D'Angelo F, Zambrano N, Terracciano $\mathrm{L}$, Iolascon $\mathrm{A}$. Changes of the hepatic proteome in hepatitis B-infected mouse model at early stages of fibrosis. J Proteome Res, 2008, 7: 2642-2653

14 Liu Y, He J, Ji S, Wang Q, Pu H, Jiang T, Meng L, Yang X, Ji J. Comparative studies of early liver dysfunction in senescenceaccelerated mouse using mitochondrial proteomics approaches. Mol Cell Proteomics, 2008, 7: 1737-1747

15 Diamond DL, Jacobs JM, Paeper B, Proll SC, Gritsenko MA, Carithers RL Jr, Larson AM, Yeh MM, Camp DG 2nd, Smith RD, Katze MG. Proteomic profiling of human liver biopsies: hepatitis $\mathrm{C}$ virus-induced fibrosis and mitochondrial dysfunction. Hepatology, 2007, 46: 649-657

16 Mölleken C, Sitek B, Henkel C, Poschmann G, Sipos B, Wiese S, Warscheid B, Broelsch C, Reiser M, Friedman SL, Tornøe I, Schlosser A, Klöppel G, Schmiegel W, Meyer HE, Holmskov U, Stühler K. Detection of novel biomarkers of liver cirrhosis by proteomic analysis. Hepatology, 2009, 49: 1257-1266

17 Teufelhofer O, Parzefall W, Elbling L, Kainzbauer E, Grasl-Kraupp B, Zielinski C, Schulte-Hermann R, Gerner C. Divide and conquer: rat liver tissue proteomics based on the analysis of purified constituents. Electrophoresis, 2006, 27: 4112-4120

18 Hirsch J, Hansen KC, Choi S, Noh J, Hirose R, Roberts JP, Matthay MA, Burlingame AL, Maher JJ, Niemann CU. Warm ischemia-induced alterations in oxidative and inflammatory proteins in hepatic Kupffer cells in rats. Mol Cell Proteomics, 2006, 5: 979-986

19 Li X, Xie C, Cao J, He Q, Cao R, Lin Y, Jin Q, Chen P, Wang X, Liang $\mathrm{S}$. An in vivo membrane density perturbation strategy for identification of liver sinusoidal surface proteome accessible from the vasculature. J Proteome Res, 2009, 8: 123-132

20 Kristensen DB, Kawada N, Imamura K, Miyamoto Y, Tateno C, Seki S, Kuroki T, Yoshizato K. Proteome analysis of rat hepatic stellate cells. Hepatology, 2000, 32: 268-277

21 Zhang L, Peng X, Zhang Z, Feng Y, Jia X, Shi Y, Yang H, Zhang Z, Zhang X, Liu L, Yin L, Yuan Z. Subcellular proteome analysis unraveled annexin A2 related to immune liver fibrosis. J Cell Biochem, 2010, 110: 219-228

22 Parzefall W, Kainzbauer E, Qin HM, Chabicovsky M, SchulteHermann R. Response of isolated hepatocytes from carcinogen sensitive $(\mathrm{C} 3 \mathrm{H})$ and insensitive $(\mathrm{C} 57 \mathrm{BL})$ mice to signals inducing replication or apoptosis. Arch Toxicol, 2002, 76: 699-706

23 Friedman SL, Roll FJ. Isolation and culture of hepatic lipocytes, Kupffer cells, and sinusoidal endothelial cells by density gradient centrifugation with Stractan. Anal Biochem, 1987, 161: 207-218

24 Wu J, Ma H, Qu Q, Zhou WJ, Luo YP, Thangaraj H, Lowrie DB, Fan $\mathrm{XY}$. Incorporation of immunostimulatory motifs in the transcribed region of a plasmid DNA vaccine enhances Th1 immune responses and therapeutic effect against Mycobacterium tuberculosis in mice. Vaccine, 2011, 29: 7624-7630

25 Zhang LJ, Wang XE, Peng X, Wei YJ, Cao R, Liu Z, Xiong JX, Yin XF, Ping C, Liang S. Proteomic analysis of low-abundant integral plasma membrane proteins based on gels. Cell Mol Life Sci, 2006, 63: 1790-1804

26 Zhang L, Jia X, Zhang X, Sun J, Peng X, Qi T, Ma F, Yin L, Yao Y, Qiu C, Lu H. Proteomic analysis of PBMCs: characterization of potential HIV-associated proteins. Proteome Sci, 2010, 8: 12

27 Scoazec JY, Feldmann G. In situ immunophenotyping study of endothelial cells of the human hepatic sinusoid: results and functional implications. Hepatology, 1991, 14: 789-797

28 Couvelard A, Scoazec JY, Feldmann G. Expression of cell-cell and cell-matrix adhesion proteins by sinusoidal endothelial cells in the normal and cirrhotic human liver. Am J Pathol, 1993, 143: 738-752

29 Ishihama Y, Oda Y, Tabata T, Sato T, Nagasu T, Rappsilber J, Mann M. Exponentially modified protein abundance index (emPAI) for estimation of absolute protein amount in proteomics by the number of sequenced peptides per protein. Mol Cell Proteomics, 2005, 4: 1265-1272

30 Shinoda K, Tomita M, Ishihama Y. emPAI Calc-for the estimation of protein abundance from large-scale identification data by liquid 
chromatography-tandem mass spectrometry. Bioinformatics, 2010, 26: $576-577$

31 Malhi H, Gores GJ. Cellular and molecular mechanisms of liver injury. Gastroenterology, 2008, 134: 1641-1654

32 Aroor AR, Roy LJ, Restrepo RJ, Mooney BP, Shukla SD. A proteomic analysis of liver after ethanol binge in chronically ethanol treated rats. Proteome Sci, 2012, 10: 29

33 Malhi H, Gores GJ, Lemasters JJ. Apoptosis and necrosis in the liver: a tale of two deaths? Hepatology, 2006, 43: S31-44

34 Serviddio G, Bellanti F, Sastre J, Vendemiale G, Altomare E. Targeting mitochondria: a new promising approach for the treatment of liver diseases. Curr Med Chem, 2010, 17: 2325-2337

Open Access This article is distributed under the terms of the Creative Commons Attribution License which permits any use, distribution, and reproduction in any medium, provided the original author(s) and source are credited.

\section{Supporting Information}

Figure S1 Histopathology of liver fibrosis examined by Masson's trichrome staining. (A-D, 100x) Nor, saline-treated rats livers at 2, 4, 6, and 8 weeks (W); ILF, $(\mathrm{E}-\mathrm{H}, 100 \times)$, pig serum-treated rat livers at $2,4,6$, and $8 \mathrm{~W}$. No obvious difference was found at $2-8 \mathrm{~W}$ in normal rats. In the ILF model, the degree of liver fibrosis was increased gradually from S0-1 at $2 \mathrm{~W}, \mathrm{~S} 2$ at $4 \mathrm{~W}$, and S3-4 at $6 \mathrm{~W}$ to S4 at $8 \mathrm{~W}$ as highlighted in green and indicated by arrowheads.

Figure S2 H\&E staining to monitor the purity of isolated hepatic NPCs. A, H\&E staining of the hepatic parenchymal cell fraction, 100×. B, H\&E staining of the NPC fraction purified by Percoll density gradient centrifugation, 100×. C and D, H\&E staining of the NPC fraction, 400×. 1, hepatocytes; 2, NPCs (lymphocytes); 3, NPCs (fibrocytes); 4, NPCs (KCs); 5, red blood cells.

Figure S3 Validation of the enrichment of NPCs by flow cytometry. F4/80, CD146, and CD3 were used to detect LSECs, KCs, and lymphocytes, respectively. CD3-positive cells accounted for $30.3 \%$ of NPCs and $18.7 \%$ of total liver cells (B and D). F4/80-positive cells accounted for 8.24\% of NPCs and $2.28 \%$ of total liver cells (F and H). CD146-positive cells accounted for $42.4 \%$ of NPCs and $10.4 \%$ of total liver cells (J and L). Unstained cells were used as negative controls as shown in A and C for CD3, E and G for F4/80, and I and K for CD146.

Figure S4 Protein expression profiles of the ILF model and normal control. Twelve raw 2DE gel images labeled with differential spots are shown. ILF, immune liver fibrosis; Nor, normal control. Up-regulated proteins are labeled in ILF gels and down-regulated proteins are labeled in Nor gels.

Figure S5 MS/MS spectrum of the protein (carboxylesterase 3) identified by one peptide.

Figure S6 Protein-protein interaction network identified by STRING software using differentially expressed proteins as seeds.

Figure S7 qRT-PCR analysis of the eight selected genes in controls at 8 weeks. GAPDH was used as an internal standard. A, qRT-PCR amplification curve. B, Ct values (threshold cycle). C, Standard curve. D, Melting curve. The eight target genes could be amplified and quantified by qRT-PCR. There was no detection of non-specific PCR products.

Table S1 Raw data of ImageMaster software analysis. Intensity value, volumes, volume\% (after normalization), area of identified differential spots, and the numbers of matched protein spots in each gel are shown

Table S2 The peptides of differentially expressed proteins

Table S3 The relative mRNA expression in ILF models compared with that in normal controls at 4 and 8 weeks as determined by qRT-PCR

The supporting information is available online at life.scichina.com and link.springer.com. The supporting materials are published as submitted, without typesetting or editing. The responsibility for scientific accuracy and content remains entirely with the authors. 